\title{
Research and Software Implementation of PLC Channels Simulation System Based on FPGA
}

\author{
Liu Wei ${ }^{1, a}$, Zhang Xue ${ }^{2, b}$, Qingyang Liu $^{2, c}$ \\ ${ }^{1}$ STATE GRID ANSHAN ELECTRIC POWER SUPPLY BRANCH \\ An Shan, China \\ ${ }^{2}$ NARI Group, CHINA GRIDCOM CO,.LTD \\ Shen Zhen, China \\ ${ }^{a}$ E-mail: 13358658181@189.com, ${ }^{b}$ E-mail: zhangxue2@sgepri.sgcc.com.cn, \\ cE-mail: liuqingyang@sgepri.sgcc.com.cn
}

Keywords: PLC; noise; Channel Frequency Response; Field Programmable Gate Array (FPGA); Simulation; Filter, Communication performance evaluation

\begin{abstract}
Power line communication (PLC) has shown great advantages in the smart grid, broadband access, intelligent home and four nets fusion fields.But its bad channel environment especially the noise interference problem is so serious that has become a bottleneck restricting of the development.In this paper,we present a simulation system with five different access channels,four indoor channel parameter models and five kinds of typical power line noise models and its software implementation based on FPGA ,by doing research on the characteristics of power line channel and channel noises.And built the hardware simulation and test platform for system testing. To lay foundation of real-time simulation and evaluation for different PLC devices communication performance in different channel environment and noise environment.
\end{abstract}

\section{Introduction}

Power Line Communication (PLC) refers to the communication method that using the existed power cables to transfer data,voice, image and other multimedia information. It has broad application prospects for the advantages: without re wiring, wide covering range and convenient connection. However, the power line is a time-varying system with complex network structure, the channel showed the characteristic: impedance variation, unstable channel frequency characteristics,strong signal interference and large time-varying. Therefore, the frequency response and noise characteristics of power line channel are simulated, and it can advance to simulate and test the communication performance of communication equipment in different channel environments and different types of noise environment.and make the hardware or coding which used to improve the communication quality more anticipation, pertinence and feasibility.This paper proposed a design scheme of the power line channel simulation system based on FPGA, and tested this simulation system by building a hardware test platform.

\section{Simulation of Power Line Channel}

Channel characteristics of power line network are different from other communication networks such as: noise characteristics, impedance characteristic, attenuation characteristic and multipath characteristics. The characteristics of the power line channel are the key research in power line communication system. In order to make the PLC device adapt to the power line communication network in China better, we 
need to know the channel characteristics accurately and establish the corresponding channel model for further theoretical analysis of power line channel characteristics.

Many domestic and foreign experts have done lots of conducted field tests on power line channel, and have simulated all kinds of different channel models with computers .In general, these models can be roughly divided into the following two categories: The first method of modeling is from top to bottom, the relevant parameters to build the channel model is extracted through the method of various parameter fitting from the field measurements, and analyze a general mathematical model of power line channel that frequency range is $500 \mathrm{kHz} 30 \mathrm{MHz}$; The second one is bottom-up modeling, the power line network is regarded as a two-port network, using a large number of discrete components to build the entire network, and establish the power line channel model by solving the transmission matrix.

In this paper we proposed the noise simulation module with 5 kinds of typical noise and the channel simulation module with 4 indoor channel and 5 types of access channel based on the power line channel simulation and channel noise simulation theory research, and verify the correctness of each module through MATLAB simulation. The channel simulation module and noise module are integrated in the engineering in FPGA simulation software to form a complete system, and measure this system by building a hardware test platform .

\section{Simulation System Design}

The system consists of industrial personal computer (IPC), FPGA board, AD/DA boards, and realized the simulation of the channel frequency response and channel noise by FPGA control.Loading the channel simulation module and noise module in FPGA, the communication status in different channels and different noises can be simulated and displayed while the parameters of each module configuration is selected and controlled by the IPC.The PLC signal is sampled by AD board which converting the analog signal into digital signal ,and the digital signal is transmitted to the FPGA for processing in the digital domain,the processed digital signal is converted into analog signal to output by DA card. The simulation system design diagram as shown in figure 1.

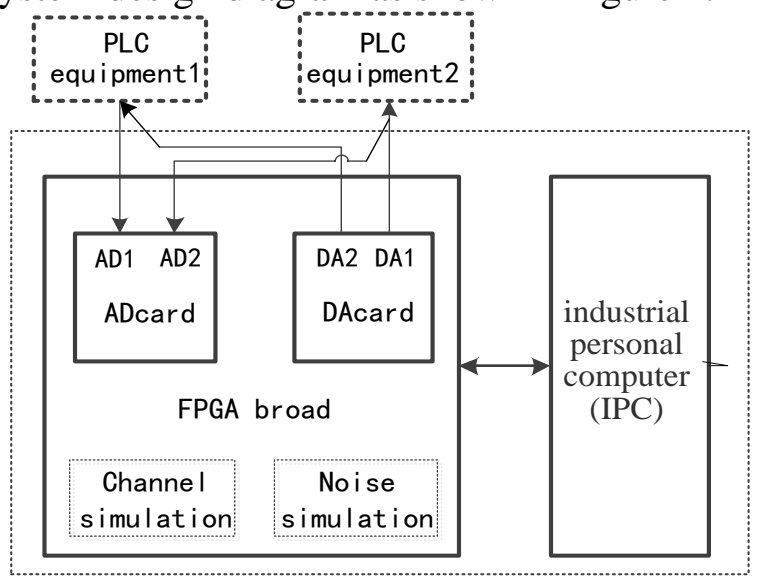

Figure 1. Diagram of simulation system design

A. Simulation of frequency response characteristic of power line channel

- Software design of channel simulation

Channel simulation system is consists of indoor channel module, channel access module and a custom channel module.Indoor reference channels have notch characteristics, and are divided into four types according to different degree of attenuation of channel in frequency domain.Access reference channel module have notch characteristics and low pass filter characteristic, and are divided into five 
types according to different degree of channel attenuation in frequency domain. channel selection are all through the channel configuration options in each module.However custom channel complete the channel allocation according to the frequency response function of channel to obtain the filter tap coefficients and delay in PC or other equipment.

- $\quad$ FIR filter realization principle

FIR finite impulse response filter is a digital filter, its unit impulse response is a sequence with finite length, so the system function can be expressed:

$$
H(z)=\sum_{n=0}^{N-1} h(n) z^{-n}
$$

Differential equation related to input and output of FIR filter system can be written as:

$$
y(n)=\sum_{n=0}^{N-1} h(m) x(n-m)
$$

According to the relation between input and output of the the system,the structure can be implemented in the Verilog by using the adder and multiplier. In fact, when the coefficients of filter are symmetrical, the input and output can be expressed as:

$$
y(n)=\sum_{n=0}^{(N-1) / 2} h(n)(x(n)+x(N-1-n))
$$

- Software realization

Software realization of channel simulation is to use the top-level module to specialize module features and then output one channel selectively. Indoor channels realize the function of notch filter through FIR filter with direct type,the indoor module configured four kinds of indoor channel with different degree of attenuation. Access channel assembled with a notch filter and a low-pass filter is actualized by direct FIR with notch filters and Low pass filter with symmetry respectively,the access module configured five kinds of access channel with different degree of attenuation. The custom channel module Custom_Fir configured the corresponding custom filter combined coefficient and custom filter delay combined file,channel from each module will be gated and outputted by option .

B. Noise simulation of power line channel

- introduction of typical noises

Usually, the noise of power line is divided into the following five categories:

(1)The colored background noise is mainly produced by noise source with various low power, it changes with frequency changed and has a relatively low power spectral density(PSD).

(2)Narrow band noise is generally caused by medium wave and short wave radio,it changes with time.

(3)The repetition frequency of the periodic pulse noise which is asynchronous with power frequency is generally $50 \mathrm{kHz} \sim 200 \mathrm{kHz}$.

(4)The repetition frequency of the periodic pulse noise which is synchronous with power frequency or its Integer is $50 \mathrm{~Hz}$ or $100 \mathrm{~Hz}$.

(5)Random pulse noise is produced by lightning or switch operation of the load on the network, its arrival time is random and can last from a few s to a few ms.

- Software realization of channel noise simulation

The colored background noise is produced by a pseudo random sequence m_sequence generated by white noise passing through a low pass filter. The filter adopts a adder tree with full parallel architecture that has direct type structure with 17 tap coefficient to actualize, and to realize the whole function of the colored background noise by top file.We can choose to output white noise or colored noise through option and can adjust its amplitude.Diagram of function module structure is shown as below: 


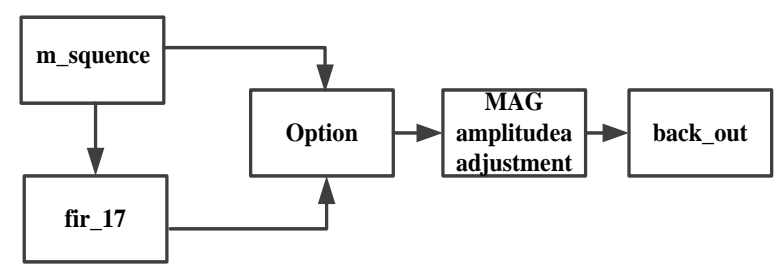

Figure 2. Function module of the background noise

Narrow band noise is generated by superposition of sine waves with varying amplitude and bandwidth,the sine waves are produce by DDS (digital frequency synthesis).DDS is composed of the internal phase accumulator and sine look-up table and the external DAC and LPF.Narrow band noise files can realize the accumulation function, custom amplitude modulation, and the transfer and accumulation function of custom frequency control.Narrow-band noise is generated by sine wave superposition while sine wave produced by DDS is completed .Diagram of the function module realization of narrow band noise is shown in Figure 3.

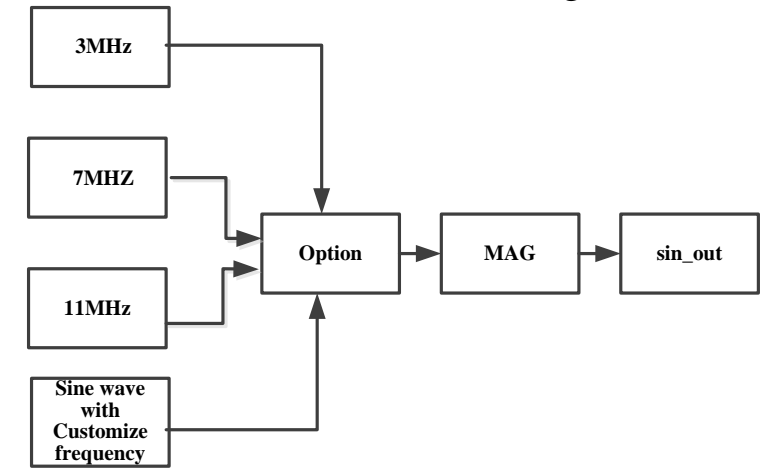

Figure 3. Function module of narrow band noise.

The periodic pulse noise synchronized with power frequency is generated by pseudo- random sequence m_sequence with switching strobe.On_off_50 and on_off_100 refer to the frequencies of 50Hz and $100 \mathrm{~Hz}$ respectively.it is chose to output by option and its amplitude is adjusted by MAG.

Random impulse noise uses Markov chain to generate random pulse by on-off switch,. ap_ medium _50k, ap_medium_100k and ap_medium_200k respectively represent periodic noise frequency of $50 \mathrm{kHz}, 100 \mathrm{kHz}$ and $200 \mathrm{kHz}$ which are asynchronous with power frequency.The noise is chose to output by option and its amplitude is adjusted by MAG.Different intensity of random noise of power line is realized by modules with different intensity in the top program diagram of the function module .

We can observed the waveform in time domain or in frequency domain and the power spectrum density after the superposition of these four kinds of noise in power line by spectrum meter or oscilloscope while the above four categories of noise are superimposed and synthesized selectively.

\section{Test Result}

A. Test results of channel frequency response characteristic simulation

Result of channel simulation used by MTALAB tested in the range of $0 \sim 35 \mathrm{MHz}$ is as shown in Figure 4and the measured drawing of channel simulation is as Figure5, the frequency we tested is in the range of $0 \sim 50 \mathrm{MHz}$,access reference channel 1 and indoor reference channel 1 shown below are choose arbitrarily. 


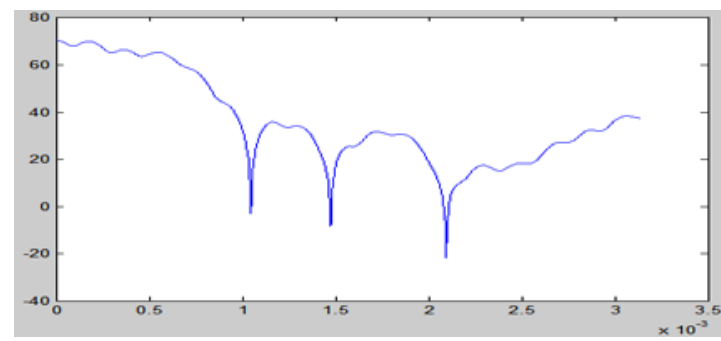

(a) access reference channel 1

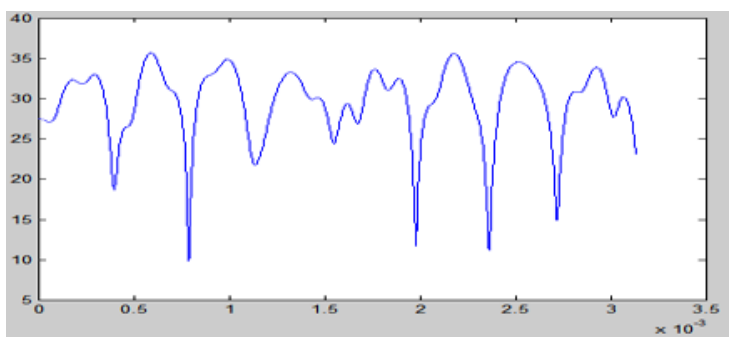

(b) indoor reference channel 1

Figure 4. Simulation results of channel simulated by MATLAB

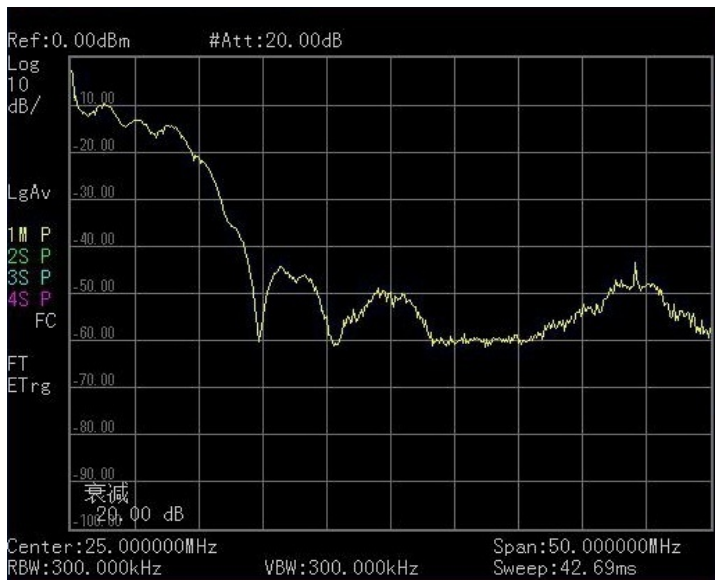

(a) access reference channel 1

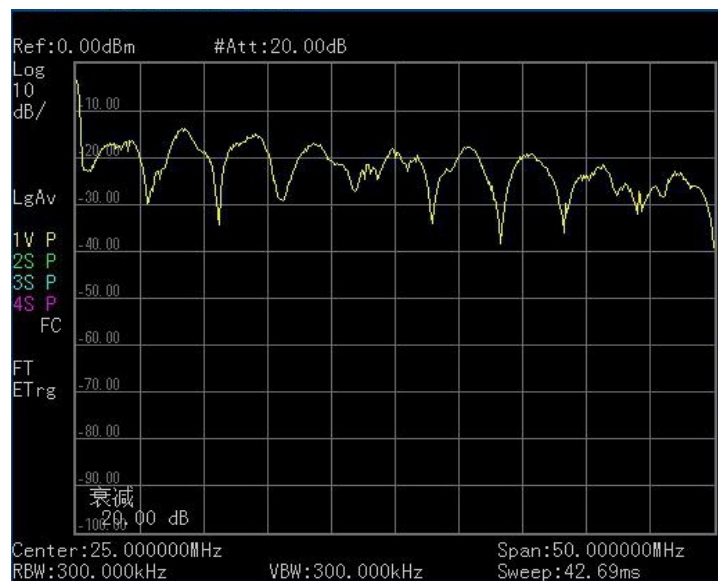

(b) indoor reference channel 1

Figure 5. Test result of channel simulation

The measurement results and simulation results using by MATLAB of access channel and the indoor channel are consistent.

B. Test results of channel noise simulation

The measured drawing of channel noise simulation is as below,the frequency we tested is in the range of $0 \sim 50 \mathrm{MHz}$,synthetic noise shown is comprised of background noise, $11 \mathrm{MHz}$ narrow band noise and Markov noise, and their intensity are $10 \mathrm{~dB} 、 24 \mathrm{~dB}$ and $24 \mathrm{~dB}$ respectively.

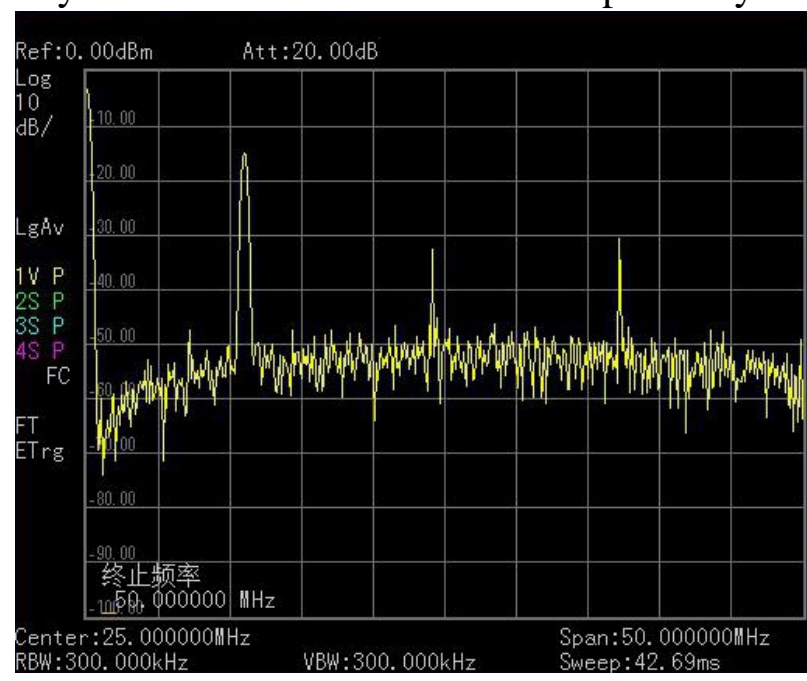

Figure 6. Channel noise synthesis simulation of power line

We can see that it not only can output single noise, but also can realize the combination 
of the noises, and this situation is consistent with the actual noises in power line. So, the channel noise simulation work is more applicable and practical.

\section{Conclusion}

This paper proposed the design idea of the power line channel simulation system based on FPGA through the study of power line channel characteristics and channel noise characteristics. And detailed the method of software realization which is use to simulate power line channel characteristics with different structure and noise characteristics in different environment through IPC to configure channel model and noise model flexibly. It has given the measurement results of channel frequency response characteristics and the channel noise simulation, that provide a basis for the communication performance evaluation of power line carrier communication device with different channel characteristics or in different noise environment.

\section{References}

[1] Gotz M, Rapp M, Dostert K. Power line channel characteristics and their effect on Communication system design[J].Communications Magazine,IEEE ,42(4):78-86 2004.

[2] Meng H, Guan Y L, Chen S. Modeling and analysis of noise effects on broadband power-line communications[J]. Power Delivery, IEEE Transactions on, 2005, 20(2): 630-637.

[3] Sancha S, Canete F J, Diez L, et al. A channel simulator for indoor power-line communications [C].Power Line Communications and Its Applications, 2007. ISPLC'07. IEEE International Symposium on. IEEE, 2007: 104-109.

[4] Zhang S Q,Yu D H.The measurement and analysis of noise characteristic in low voltage power line communication channel[J].Telecommunications for Electric Power System,2003, 24(1): 35-38.

[5] Zhang Z F,Jiang Z.Measurement and Study on indoor low-voltage power line channel.Electrical Measurement\&Instrumentation,2008 (9).

[6] Zhang Y B,Zhao Z H,Weng G Q.Real-time simulation of power line communication channels based on FPGA.Electric Power Automation Equipment.2011, 31(8): 122-126.

[7] Lin H,Lin D.Modeling of PLC Noise and Design of Noise Generator[J]. Telecommunications for Electric Power System.2012, 25(5): 34-37.

[8] Feng H. Li S Q.Design and Realization of Power Line Simulation System on FPGA[J]. Electronic science and technology.2012, 25(5): 34-37.

[9] Zhang H X,Zhang X M,Tai X.Noise analysis and modeling research in power line channel[J]. Journal of Chongqing University of Posts and Telecommunications (Natural Science Edition).ISTIC, 2013, 25(5). 